\title{
INSECT RECONNAISSANCE IN LIBERIA, WEST AFRICA
}

\author{
By M. S. Briscoe \\ College of Medicine, Howard University, \\ Washington, D. C.
}

The Republic of Liberia lies approximately between $4^{\circ} 22^{\prime}$ to $8^{\circ} 50^{\prime}$ north latitude, and $7^{\circ} 33^{\prime}$ to $11^{\circ} 32^{\prime}$ longitude west of Greenwich. It has one of the most humid and rainiest climates of Africa. The rainy season begins in April and terminates in November, the annual rainfall being as much as 170 inches.

This paper is a report of an insect reconnaissance in many localities in the hinterland and coastal areas of Liberia. Collections were made throughout the year. Accordingly, an overall picture of seasonal distribution was obtained; this will be reported in a later paper.

In the following list families are arranged in accord with recent phylogenetic studies. Among the Diptera there are five new distributional records for Liberia and four undescribed species. Definite localities are given for all of the specimens.

\section{Diptera}

Tipulidæ

1. Trentepohlia exornata Bergr. Roberts Field; Harbel.

The collection also contains unidentified species of Elephantomyia, Lecteria, Megistocera and Tipula collected at Roberts Field and Harbel.

Bibionidæ

Unidentified species of Plecia collected at Roberts Field and Harbel.

Fungivoridæ

Unidentified species of Cerotelion, Delopsis, Lycoria, Neoempheria and Trichosia collected at Roberts Field and Harbel. 
Tendipedidæ

Unidentified species of Tendipes collected at Roberts Field.

\section{Heleidæ}

Unidentified species of Forcipomyia collected at Roberts Field.

Psychodidæ

2. Brunettia albonotata (Brun.) Harbel.

3. Telmatoscopus albipunctatus (Will.) Roberts Field; Harbel.

Culicidæ

4. Anopheles funestus Giles. Roberts Field; Harbel; Gbanga.

5. Anopheles gambia Giles. Roberts Field; Harbel; Owen's Grove; Marshall; Kakata; Fisherman's Lake; Reputa; Salala; Cape Palmas; Grand Bassa.

6. Anopheles hancocki Edw. Roberts Field; Harbel; Fisherman's Lake.

7. Anopheles nili (Theob.) Roberts Field; Harbel.

8. Anopheles paludis Theob. New record for Liberia. Roberts Field.

9. Aëdes (Aëdimorphus) sp. very close to filis I. \& M. Roberts Field.

10. Aëdes agypti (L.) Roberts Field; Harbel; Kakata; Monrovia.

11. Aëdes africanus (Theob.) Roberts Field; Kakata.

12. Aëdes luteocephalus Newst. Roberts Field.

13. Aëdes vittatus (Bigot). Roberts Field.

14. Culex annulirostris consimilis Newst. Roberts Field; Harbel.

15. Culex bitaniorhynchus Giles. Roberts Field; Harbel.

16. Culex guiarti Blanch. Roberts Field; Harbel.

17. Culex moucheti Evans. Roberts Field; Harbel.

18. Culex nebulosus Theob. Roberts Field; Harbel.

19. Culex tigripes Gr. \& Ch. Roberts Field; Harbel.

20. Eretmapodites chrysogaster Graham. Roberts Field; Harbel.

21. Mansonia africana (Theob.) Roberts Field; Harbel. 
22. Mansonia metallica (Theob.) Roberts Field.

23. Mansonia uniformis (Theob.) Roberts Field.

24. Megarhinus sp. probably brevipalpis conradti Grünb. Roberts Field; Harbel.

The collection also includes unidentified species of Ficalbia (Miomyia) collected at Roberts Field.

\section{Tabanidæ}

25. Chrysops longicornis Macq. Roberts Field.

26. Tabanus besti var. arbucklei Austen. Roberts Field; Harbel.

27. Tabanus par Walk. New record for Liberia. Roberts Field.

28. Tabanus kingsleyi Ricardo. Roberts Field; Harbel.

29. Tabanus postacutus Oldroyd. New record for Liberia. Roberts Field; Harbel.

30. Tabanus taniola Palis. de B. Roberts Field.

31. Thaumastocera akwa Grünberg. New record for Liberia. Roberts Field.

32. Hamatopota furians Edw. New record for Liberia. Roberts Field.

33. Hæmatopota guineensis Bigot. Roberts Field.

Rhagionidæ

Unidentified species of Suragina collected at Roberts Field and Harbel.

\section{Asilidæ}

Two new species, Laphria and Ommatius, collected at Roberts Field.

\section{Dorilaidæ}

Unidentified species of Dorilas collected at Roberts Field and Harbel.

$$
\text { Syrphidæ }
$$

34. Baccha brevis Karsch. Roberts Field; Harbel.

35. Melanostoma annulipes Macq. var. mauritianum Big. Roberts Field; Harbel.

36. Microdon brevicornis Loew. Roberts Field.

37. Microdon punctulatus Wied. Roberts Field.

38. Paragus borbonicus Macq. Roberts Field. 
39. Protylocera asacus Walker. Roberts Field; Harbel; Kakata.

40. Protylocera dibaphus Walker. Roberts Field; Harbel; Kakata.

41. Rhingia carulescens Loew. Roberts Field; Harbel; Kakata.

The collection also contains unidentified species of Tubifera and two new species, Eumerus near triangularis Hervé-Bazin, and Graptomyza, collected at Roberts Field.

Tephritidæ

42. Ceratitis (Pardalaspis) giffardi Bezzi. Roberts Field; Harbel.

43. Ceratitis (Pardalaspis) punctata (Wiedmann). Roberts Field; Harbel.

44. Ceratitis (Trirhithrum) fraterna Munro. Roberts Field; Harbel.

45. Ceratitis (Trirhithrum) nigerrima (Bezzi). Roberts Field; Harbel.

46. Dacus armatus F. Roberts Field; Harbel.

47. Dacus punctatifrons Karsch. Roberts Field; Harbel. The collection also includes unidentified species of Rhacochlana collected at Roberts Field.

Glossinidæ

48. Glossina fusca Walker. Roberts Field; Reputa; Kolobanu.

49. Glossina nigrofusca Walker. Roberts Field; Memmeta.

50. Glossina palpalis Rob.-Desv. Roberts Field; Kolobanu; Lengatown; Banga; Reputa; Memmeta; Kakata.

Hymenoptera

Chalcidoidea

Unidentified species of Brachymeria, Cleonymus, Philomides and Ptinobius collected at Roberts Field.

Braconidæ

Unidentified species of Iphiaulax collected at Roberts Field and Harbel. 
Ichneumonidæ

51. Idechthis canescens (Grav.) Roberts Field; Harbel.

The collection also includes unidentified species of Anomalon, Charops, Enicospilus, Melanichneumon, Pristomerus, Xanthocampoplex, Xanthopimpla and Zaleptopygus collected at Roberts Field.

\section{Tiphiidæ}

52. Tiphia pedestris Gerst. Roberts Field; Harbel.

Scoliidæ

53. Campsomeris albicollis (Chr.) Roberts Field.

The collection also contains unidentified species of Scolia collected at Harbel.

\section{Mutillidæ}

54. Timulla cyparissa (Sm.) Roberts Field.

The collection also includes unidentified species of Odontomutilla collected at Roberts Field and Harbel.

Chrysididæ

55. Chrysis lyncea F. Roberts Field; Harbel ; Marshall; Kakata; Reputa.

Formicidæ

56. Dorylus (Dorylus) gribodoi Emery. Roberts Field; Harbel; Monrovia; Salala.

57. Dorylus (Anomma) nigricans Illiger. Roberts Field; Harbel; Monrovia.

58. Ecophylla longinoda var. fusca Emery. Roberts Field; Harbel; Kakata.

59. Paltothyreus tarsatus (F.) Roberts Field.

The collection also contains unidentified species of Crematogaster collected at Roberts Field and Harbel.

\section{Pompilidæ}

60. Cyphononyx croceicornis (Er.) Harbel.

61. Paracyphononyx zonatus (Ill.) Roberts Field.

62. Pseudagenia personata Grib. Roberts Field.

The collection also contains unidentified species of Anoplius collected at Roberts Field. 


\section{Vespidæ}

63. Polistes marginalis var. ornatus Lep. Roberts Field.

64. Polybioides tabida (F.) Roberts Field.

65. Ropalidia cincta (Lep.) Roberts Field.

66. Belonogaster junceus (F.) Roberts Field.

67. Rygchium marginellum (F.) Roberts Field; Harbel.

68. Rygchium albonigrum (J. Bequaert). Roberts Field; Harbel.

69. Rygchium synagroides (Sauss.) Harbel.

70. Rygchium ventrale (Sauss.) Harbel.

71. Synagris calida (L.) Harbel.

72. Synagris cornuta (L.) Roberts Field; Harbel; Marshall.

73. Synagris spiniventris (Ill.) Roberts Field; Harbel.

Sphecidæ

74. Chalybion fuscipennis (Sm.) Roberts Field; Marshall.

75. Chlorion xanthoceros (Ill.) Roberts Field; Harbel; Marshall.

76. Motes crœsus (Sm.) Roberts Field.

77. Sceliphron spirifex (L.) Roberts Field; Harbel; Marshall; Kakata; Salala.

78. Sphex, near indutus (Kohl). Roberts Field; Harbel; Marshall.

The collection also includes unidentified species of $C e r$ ceris, Dasyproctus, Hoplisoides, Trypoxylon and Bembix collected at Roberts Field. The last named specimen was also found at Kakata, Reputa and Salala.

\section{Apidæ}

79. Trigona erythra Schlett. Tototown; Salala.

80. Trigona erythra togonsis Stad. Tototown; Salala.

81. Trigona (Dactylurina) staudingeri Grib. Roberts Field; Harbel.

82. Anthophora cincta F. Roberts Field; Harbel.

83. Mesotrichia albiceps (F.) Reputa; Salala.

84. Mesotrichia gabonica Vach. Roberts Field; Harbel.

85. Mesotrichia imitator nigriceps Friese. Roberts Field. 
86. Mesotrichia modesta (Smith). Roberts Field; Harbel.

87. Mesotrichia preusta (Smith). Roberts Field.

88. Mesotrichia torrida (Westw.) Roberts Field; Harbel.

89. Pasites nigerrima Fr. Roberts Field.

90. Euaspis abdominalis (F.) Roberts Field; Harbel.

91. Gronoceras pretexta (Vach.) Roberts Field; Harbel; Marshall.

92. Megachile crocutella Ckll. Roberts Field.

93. Megachile rufipes (F.) Harbel; Reputa.

94. Pachyanthidium xanthostomum Ckll. Roberts Field.

The collection also contains unidentified species of Xylocopa, Crocisca and Halictus collected at Roberts Field and Harbel.

\section{Coleoptera}

Carabidæ

Unidentified species of Anisodactylus, Craspedophorus, Ochyropus, Orthogonus and Pheropsophus collected at Roberts Field.

\section{Hydrophilidæ}

Unidentified species of Sternolophus collected at Roberts Field.

Staphylinidæ

Unidentified species of Paderus collected at Roberts Field.

\section{Histeridæ}

Unidentified species of Hister collected at Roberts Field and Harbel.

Lycidæ

95. Lycus latissimus (L.) Roberts Field.

96. Lycus sinuatus Dalm. or semiamplexus Murray. Roberts Field.

The collection also includes unidentified species of Speckia collected at Roberts Field.

Meloidæ

Unidentified species of Zonabris and Zonitis collected at Roberts Field and Epicauta found at Reputa. 
Coccinellidæ

97. Cheilomenes axillaris Ws. Roberts Field; Harbel. 98. Chilocorus schicdtei Muls. Roberts Field; Harbel. 99. Epilachna assimilis Muls. Roberts Field; Harbel.

Tenebrionidæ

100. Odontopus cupreus F. Roberts Field.

The collection also contains unidentified species of Eupezus, Opatrinus and Prioscelis collected at Roberts Field and Harbel.

\section{Lagriidæ}

Unidentified species of Lagria collected at Roberts Field.

\section{Scarabæidæ}

101. Odoretus luteipes Cast. teste Ohaus. Roberts Field; Harbel.

102. Anomala discordabilis Dohrn. Roberts Field.

103. Anomala imarginata Ohs. Roberts Field.

104. Archon centaurus (F.) Roberts Field; Harbel.

105. Diplognatha gagates (Forst.) Harbel.

106. Heteroligus meles Bilb. Roberts Field.

107. Incala lineola Westw. Roberts Field.

108. Oryctes monocerus Ol. Roberts Field; Harbel.

109. Pachnoda marginata Drury. Roberts Field; Harbel.

110. Pachnoda tridentata (F.) Roberts Field; Harbel.

The collection also includes unidentified species of Apogonia, Eulepida and Trochalus collected at Roberts Field and Harbel.

\section{Lucanidæ}

Unidentified species of Prosopocoilus collected at Roberts Field.

\section{Cerambycidæ}

111. Acanthophorus (Tithoes) palini Hope. Owen's Grove.

112. Monochamus ruspator (F.) Owen's Grove.

113. Sternotomus regalis (F.) Roberts Field. 
The collection also contains unidentified species of Callichroma and Euporus collected at Roberts Field.

Chrysomelidæ

114. Callispa sp. near nigripes Baly. Roberts Field.

115. Cryptocephalus sp. perhaps dregei Suffr. Roberts Field.

116. Galerucella geniculata Harold. Roberts Field.

The collection also includes unidentified species of Aspidomorpha, Hispa and Monolepta collected at Roberts Field and Harbel.

\section{Anthribidæ}

117. Gynandrocerus antennalis Lac. Harbel.

118. Polycorynus sp. probably compressicornis F. Roberts Field.

The collection also includes unidentified species of Phlotrogus collected at Roberts Field.

\section{Curculionidæ}

119. Rhynchophorus phœniciensis F. Roberts Field; Harbel.

120. Sipalinus guineensis (F.) Roberts Field.

121. Temnoschoita quadripustulata (F.) Roberts Field. The collection also contains unidentified species of Lixus, Mechistocerus and Protocylas collected at Roberts Field.

Orthoptera

Blattidæ

122. Leucophca grandis (Sauss.) Roberts Field.

123. Periplaneta australasia (F.) Roberts Field; Harbel.

Gryllidæ

124. Gryllotalpa africana Beauv. Roberts Field; Harbel.

Dermaptera

Forficulidæ

125. Diaperasticus erythrocephalus (Oliv.) Roberts Field; Harbel. 
Labiduridæ

Unidentified species of Eurollia collected at Roberts Field.

\author{
IsOptera
}

Termitidæ

126. Macrotermes bellicosus (Smeathman). Roberts Field; Kakata; Salala ; Reputa ; Harbel.

Siphonaptera

Pulicidæ

127. Ctenocephalides canis (Curtis). Roberts Field; Kakata; Harbel; Monrovia.

\title{
Neuroptera \\ Myrmeleonidæ
}

128. Palpares manicatus Rambur. Roberts Field; Harbel.

\begin{tabular}{|c|c|c|c|c|c|}
\hline \multirow[b]{2}{*}{ Orders } & \multicolumn{3}{|c|}{ SUMMARY TABLE } & \multirow[b]{2}{*}{$\begin{array}{l}\text { Deter- } \\
\text { mined }\end{array}$} & \multirow[b]{2}{*}{$\begin{array}{l}\text { Undeter- } \\
\text { mined }\end{array}$} \\
\hline & Families & Genera & Species & & \\
\hline Diptera ......................... & $14^{*}$ & 43 & 71 & 50 & 21 \\
\hline Hymenoptera ....... & 12 & 55 & 69 & 44 & 25 \\
\hline Coleoptera ................. & 15 & 55 & 56 & 27 & 29 \\
\hline Orthoptera ................. & 2 & 3 & 3 & 3 & 0 \\
\hline Dermaptera ............. & 2 & 2 & 2 & 1 & 1 \\
\hline Isoptera & 1 & 1 & 1 & 1 & 0 \\
\hline Siphonaptera ....... & 1 & 1 & 1 & 1 & 0 \\
\hline Neuroptera & 1 & 1 & 1 & 1 & 0 \\
\hline 8 & 48 & 161 & 204 & 128 & 76 \\
\hline
\end{tabular}

Acknowledgments

The writer is indebted to Mr. C. F. W. Muesebeck and staff of the Bureau of Entomology and Plant Quarantine for aid in determining the specimens. Dr. Alan Stone determined the Culicidæ. Dr. Joseph Bequaert, Curator of Insects, Museum of Comparative Zoology, Harvard College, revised the Tabanidæ and Vespidæ. 

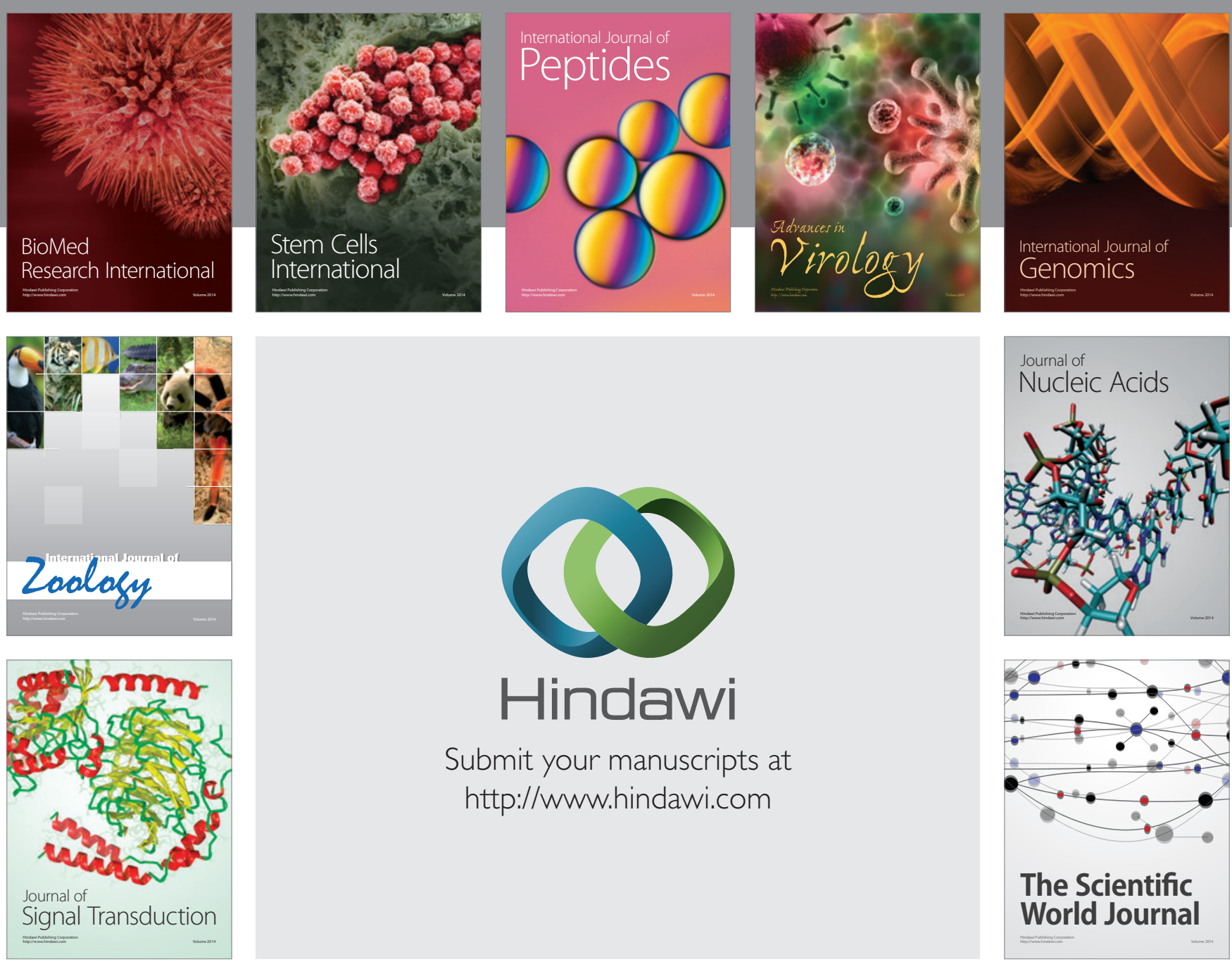

Submit your manuscripts at

http://www.hindawi.com
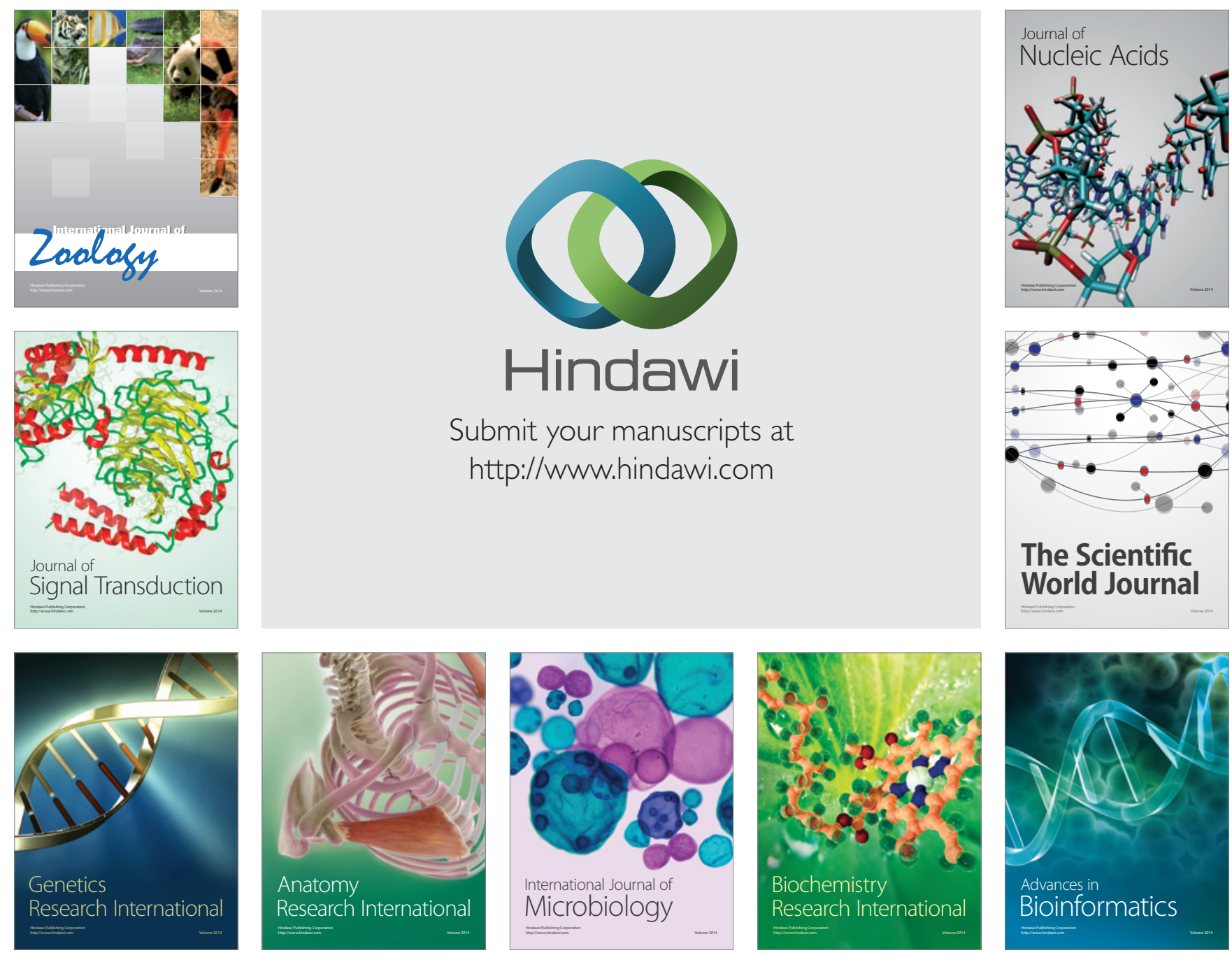

The Scientific World Journal
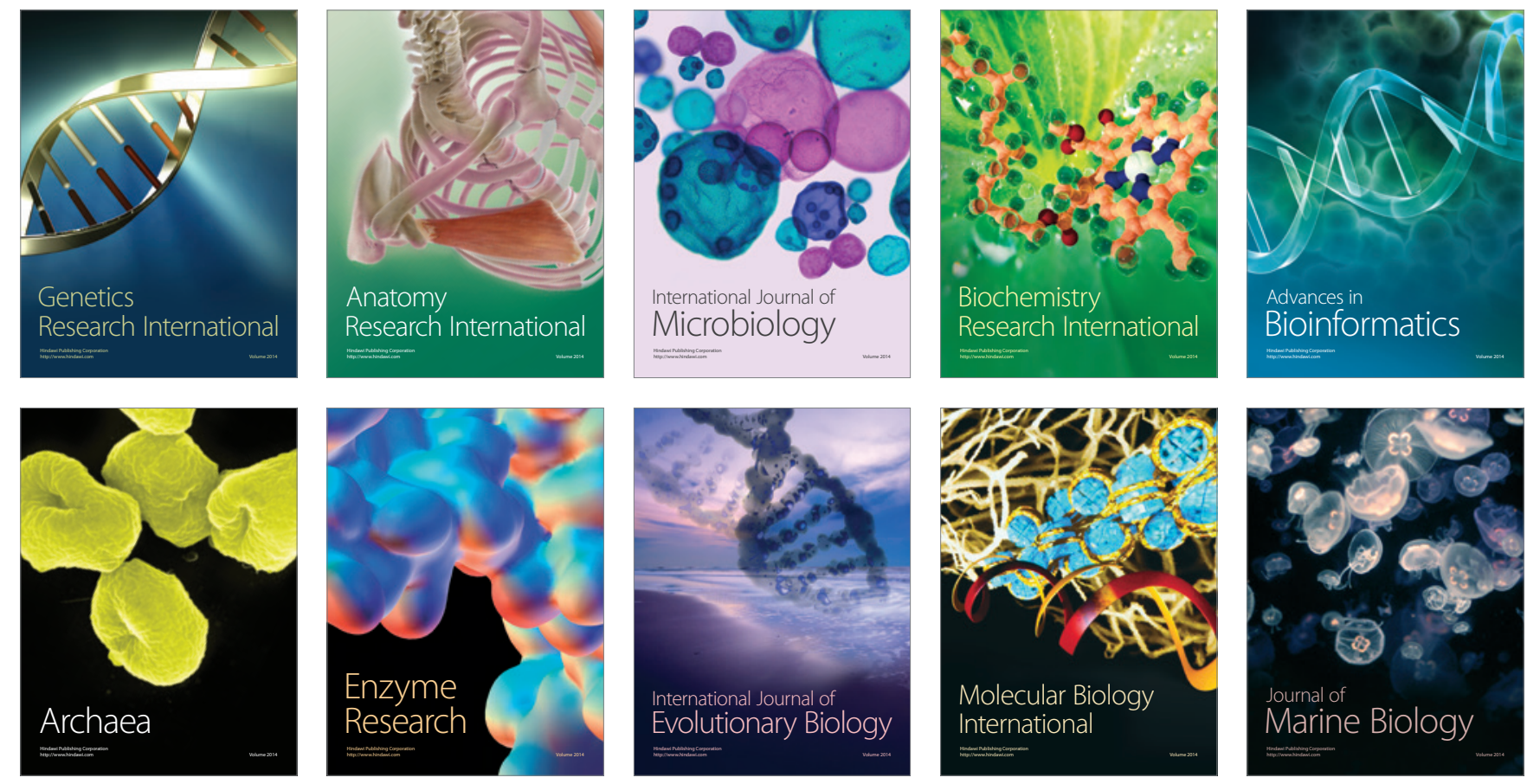